\title{
Médiévales
}

Langues, Textes, Histoire

77 | automne 2019

Mathématiques. Savoirs et enseignements (OrientOccident)

\section{L'unité et le nombre dans les Questiones mathematice de Raoul le Breton}

Unity and Number in the Questiones mathematice by Radulphus Brito

\section{Sabine Rommevaux-Tani}

\section{OpenEdition}

\section{Journals}

Édition électronique

URL : https://journals.openedition.org/medievales/10472

DOI : $10.4000 /$ medievales. 10472

ISSN : $1777-5892$

Éditeur

Presses universitaires de Vincennes

Édition imprimée

Date de publication : 5 décembre 2019

Pagination : 111-127

ISBN : 978-2-37924-061-4

ISSN : 0751-2708

Référence électronique

Sabine Rommevaux-Tani, "L'unité et le nombre dans les Questiones mathematice de Raoul le Breton », Médiévales [En ligne], 77 | automne 2019, mis en ligne le 01 janvier 2022, consulté le 24 avril 2022. URL : http://journals.openedition.org/medievales/10472 ; DOI : https://doi.org/10.4000/medievales 10472 
Sabine Rommevaux-Tani

\section{L'unité et le nombre dans les Questiones mathematice de Raoul le Breton}

Né vers 1270/73 à Ploudiry, en Bretagne, Raoul le Breton ou Radulphus Brito est maitre ès arts à Paris vers 1290. Il commence ses études de théologie en 1299, alors qu'il enseigne à la faculté des arts ; il devient docteur en théologie en 1313-1314. Il est proviseur du collège de Sorbonne de 1312-1315 à 1319. Il meurt en 1320-1321 ${ }^{1}$.

Raoul le Breton nous a laissé un commentaire des Sentences de Pierre Lombard, nécessaire à l'obtention de son doctorat de théologie. Mais les textes qui nous ont été transmis sont avant tout le reflet de son enseignement à la faculté des arts de Paris ${ }^{2}$. Dans le domaine de la philosophie, il a surtout commenté Aristote, entre autres le De Anima, la Métaphysique, la Physique, les Météorologiques. Néanmoins on connaît avant tout Raoul le Breton pour ses réflexions en logique et en grammaire, que l'on trouve en particulier dans ses commentaires de Priscien, Boèce ou encore Aristote (les Topiques et les Analytiques). Comme grammairien, il s'inscrit dans le cercle des « modistes », ce groupe d'universitaires qui voulurent faire de la grammaire une science, une «grammatica speculativa », indépendante de la logique ${ }^{3}$.

1. On trouve les rares informations biographiques sur Raoul le Breton dans J.-L. DeUfFIC, «Un logicien renommé, proviseur de Sorbonne au XIV siècle. Raoul le Breton de Ploudiry. Notes bio-bibiographiques », Pecia. Ressources en médiévistiques, 1 (2002), p. 45-154; W. C. Courtenay, « Radulphus Brito, Master of Arts and Theology », Cahiers de l'Institut du Moyen Âge grec et latin, 76 (2005), p. 131-158. Elles sont reprises dans A. M. MorAMárquez et I. Costa, « Radulphus Brito », dans E. N. Zalta éd., The Stanford Encyclopedia of Philosophy, Spring 2018 Edition, [en ligne] https://plato.stanford.edu/archives/spr2018/ entries/radulphus-brito/ [consulté le 17 juillet 2019].

2. La liste de ses œuvres se trouve dans A. M. MorA-Márquez et I. Costa, « Radulphus Brito...»

3. I. Rosier-CATACH, La Grammaire spéculative des modistes, Lille, 1983. 
Nous nous intéressons ici à ses Questiones mathematice, comme les nomme Olga Weijers ${ }^{4}$. Celles-ci se trouvent dans deux manuscrits ${ }^{5}$ : l'un à Paris, BnF, lat. 16609 (noté P), daté de 1299, soit très proche de la date de rédaction des Questiones mathematice qui se situe après $1295^{6}$; l'autre à Bruxelles, Bibliothèque royale de Belgique, 3540/47 (catalogue : 2910), daté $\mathrm{du} \mathrm{Xv}^{\mathrm{e}}$ siècle (noté $\mathrm{B}$ ). Il y a parfois des différences importantes entre les deux textes. Il est probable que le manuscrit de Bruxelles contient une version remaniée de ces Questiones.

Dans le manuscrit de Paris, les Questiones de Raoul le Breton se trouvent entre les folios 30 et 40 et sont précédées de questions anonymes sur les Seconds analytiques d'Aristote, qui se terminent avec la mention du copiste Richard d'Étréville, dit Hérout, et la date, 3 février 1299. Les Questiones de Raoul sont suivies de questions anonymes sur le De sphera de Johannes de Sacrobosco (sans doute de Raoul de Hotot ${ }^{7}$ ), de questions anonymes sur le De anima et enfin de questions anonymes sur la Physique d'Aristote (peut-être de Henri de Gand) ${ }^{8}$. Les Questiones mathematice de Raoul le Breton se trouvent donc dans un recueil de textes universitaires, principalement des commentaires d'Aristote. L'auteur est explicitement mentionné dans le colophon : «Expliciunt questiones super algorismum et compotum et questiones communes mathematice et etiam geometrice a britone date per copiam. » Le manuscrit de Bruxelles contient quant à lui exclusivement des textes de Raoul le Breton ${ }^{9}$, pour certains dans des secondes versions.

Le manuscrit de Paris contient 43 questions ; la 34 e , sur la définition de l'angle, manque dans le manuscrit de Bruxelles ${ }^{10}$.

4. O. WeIJers, La « Disputatio » à la Faculté des arts de Paris (1200-1350 environ), Turnhout, 1995,p. 161.

5. Ces deux manuscrits sont décrits dans F. HenTSCHEL, Sinnlichkeit und Vernunft in der mittelalterlichen Musiktheorie, Stuttgart, 2000, p. 281.

6. F. Hentschel et M. Pickavé, «Questiones mathematicales. Eine Textgattung der Pariser Artistenfakultät im frühen 14. Jarhundert », dans J. A. AERTSEn, K. EMERY, A. SPEER éd., Nach der Verurteilung von 1277 Philosophie und Theologie an der Universität von Paris im letzten Viertel des 13. Jahrhunderts. Studien und Texte, Berlin/New York, 2001, p. 618634 (p. 621).

7. P. GloRIEuX, Répertoire des maîtres de théologie de Paris au $\mathrm{XIII}^{\mathrm{e}}$ siècle, Paris, 1933, vol. 1, p. 453-456.

8. F. Hentschel et M. PicKavé, «Questiones mathematicales... », p. 623.

9. Ibid., p. 623.

10. Pour la liste de ces questions, voir O. WeiJers, La «Disputatio »..., p. 168-171 (il y a quelques erreurs de transcription dans les intitulés. O. Weijers inclut dans les questions de Raoul le Breton celles sur le De sphera). Franz Hentschel a publié les questions 7 (Utrum sint tantum quattuor scientia mathematica), 8 (Utrum astrologia et musica sint magis naturales quam mathematica) et les questions sur la musique. Je prépare l'édition de l'ensemble des questions dans le cadre d'un projet collectif international d'édition des œuvres complètes de Raoul le Breton : voir University of Gothenburg, Radulphi Britonis Opera, [en ligne] 


\section{Description générale des Questiones mathematice}

Les Questiones mathematice s'ouvrent sur un prologue, qui commence par des considérations générales sur l'utilité de faire de la philosophie pour atteindre la liberté et une vie bonne ${ }^{11}$. Le sujet principal de ce prologue est toutefois la division des sciences. Raoul le Breton distingue les sciences pratiques, dont la finalité est le besoin, des sciences spéculatives, dont la finalité est la vérité et qui concourent à la félicité des humains ${ }^{12}$. Les sciences spéculatives sont elles-mêmes divisées entre les sciences spéculatives auxiliaires (adminiculative), que sont la grammaire et la logique, et les sciences spéculatives principales ${ }^{13}$, les premières venant en soutien aux secondes. Les sciences spéculatives principales sont au nombre de trois : la philosophie naturelle, les mathématiques et la science divine ${ }^{14}$ ou la science des étants séparés. Les mathématiques sont présentées comme « la science des étants abstraits des sensibles par l'intellect, étants qui sont cependant conjoints aux sensibles quant à leur être ${ }^{15} »$. Parmi les mathématiques, Raoul distingue les sciences introductives qui sont « requises pour la connaissance des sciences mathématiques spéculatives principales ${ }^{16} \gg$. Il cite plusieurs manuels à ce propos : un Algorismus, un De sphera et un Computus, faisant sans doute allusion aux traités que Sacrobosco rédigea alors qu'il enseignait à la faculté des arts de Paris, dans les années 1220-1235. Raoul évoque aussi un Quadrans, renvoyant soit à l'ouvrage de Sacrobosco sur ce sujet, soit à celui de Robertus Anglicus. Enfin, Raoul parle de tabule, soit de tables astronomiques, dont l'usage

https://flov.gu.se/english/research/research-programs/topica-project/collaboration/radulphibritonis-opera [consulté le 29 juin 2019].

11. Ce prologue a été édité par O. WeIJers, La «Disputatio »..., p. 162-167.

12. Ibid., p. 163 : «Ista autem philosophia est duplex, scilicet practica et speculativa. Verumptamen in actu speculative consistit humana felicitas, ideo circa divisionem speculative insistamus. Unde speculativa scientia cuius finis est veritas, sed finis practice est opus. » (« Et cette philosophie est double, à savoir pratique et spéculative. Mais, puisque c'est de la spéculative que dépend la félicité humaine, nous insistons sur la division de la spéculative. Donc, la science spéculative est celle dont la fin est la vérité et la fin de la pratique est le besoin. »)

13. Ibid., p. 163 : «Speculativa ergo dividatur : nam quedam sunt scientie speculative adminiculative et quedam principales. Adminiculative sunt gramatica et logica. »

14. Ibid., p. 165 : «Sed scientie speculative principales sunt tres, [...], scilicet naturalis, mathematica et divina. »

15. Ibid., p. 165 : «Alia autem est scientia principalis speculativa scilicet mathematica, et dicitur quod ista est de entibus secundum intellectum abstractis a sensibilibus que tamen secundum esse sensibilibus sunt coniuncta [...]. »

16. Ibid., p. $166:$ «[...] iste mathematice introductive, sicut algorismus, spera, compotus, astralibium, quadrans et tabule, ista sunt solum quedam introductoria ordinata ad cognitionem scientiarum mathematicarum principalium speculativarum [...]. » 
était enseigné à l'aide de la Theorica planetarum Gerardi ${ }^{17}$. À côté des sciences introductives, les sciences principales sont au nombre de quatre : les deux disciplines pures que sont l'arithmétique et la géométrie et les deux disciplines en partie mathématiques et en partie physiques, que sont l'astrologie ou l'astronomie et la musique ${ }^{18}$.

Faisant suite à ce prologue, les questions 1 à 6 sont consacrées aux mathématiques en général. Raoul le Breton se demande si les choses mathématiques (mathematicalia) sont abstraites des sensibles, puis si elles sont premières par rapport aux qualités sensibles, et enfin si elles sont conjointes à ces qualités. Ayant admis que les choses mathématiques peuvent bien être abstraites des qualités sensibles, il se demande si on peut en avoir une science. Il cherche alors à circonscrire le domaine couvert par les mathématiques en demandant si celles-ci peuvent concerner les substances ou les qualités.

Les questions 7 à 10 portent sur le quadrivium, soit l'arithmétique, la géométrie, la musique et l'astronomie. Dans un premier temps, Raoul le Breton se demande s'il y a bien quatre sciences mathématiques, ni plus, ni moins. Il s'interroge alors sur le statut de la musique et de l'astrologie : relèvent-elles davantage de la philosophie naturelle que des mathématiques? Il suit alors l'opinion d'Averroès, pour qui « la musique (non pas la musique pratique mais la spéculative) et l'astronomie (non pas celle des jugements mais la spéculative) sont avant tout mathématiques, du fait qu'une science doit être dénommée à partir de ses principes plutôt qu'à partir de ses conclusions, puisque les principes sont les causes des conclusions. Or les principes de ces deux sciences sont mathématiques ${ }^{19}$ ». Raoul se demande ensuite s'il existe une mathématique commune à ces quatre sciences. Il répond que non, que la seule discipline qui puisse porter sur la quantité tant discrète que continue est la métaphysique ${ }^{20}$. Il cherche enfin à ordonner les quatre disciplines mathématiques spéculatives ; il se demande donc

17. Les manuels de mathématiques utilisés à l'université de Paris sont évoqués par G. Beaujouan, « Le quadrivium à la Faculté des arts », dans O. Weijers et L. Holtz éd., L'Enseignement des disciplines à la Faculté des arts (Paris et Oxford, $\mathrm{XIII}^{\mathrm{e}}-\mathrm{XV}^{\mathrm{e}}$ siècles), Turnhout, 1997, p. 185-194 (p. 191-192).

18. O. WeIJERS, La «Disputatio »...,p. 166: «Unde mathematice principales sunt quatuor, scilicet due pure mathematice et due partim mathematice et partim naturales ; geometria et arismetica sunt pure mathematice, sed astrologia et musica sunt partim mathematice et partim naturales. »

19. F. Hentschel, Sinnlichkeit..., p. 289 : «Verumtamen ego sustineo opinionem Commentatoris et dico, quod musica (non practica musicæ sed speculativa) et astrologia (non illa, quæ est de iudiciis, sed speculativa) sunt magis mathematicæ quam naturales, quia scientia debet denominari a principiis suis magis quam a conclusionibus, quia principia sunt causa conclusionum. Modo principia istarum duarum sunt mathematica [...]. »

20. Ms. P, f. 34ra : « Ad istam questionem dico duo. Primo quod est aliqua scientia communis ad 4 mathematicas. Secundo dico quod ista non est mathematica sed magis metaphysica. » 
laquelle de l'arithmétique ou de la géométrie est première. Sur ce point il suit Boèce, qui prouve au début de son Institution arithmétique (I, 1,9) que l'arithmétique est première par rapport à la géométrie, «car ce qui permet de faire connaître plusieurs espèces de grandeurs ou toutes est premier par rapport à la grandeur et est plus connu, or les nombres font connaître plusieurs espèces de grandeurs, comme le triangle est nommé à partir de 3 , le rectangle à partir de 4 , le pentagone à partir de 5 et ainsi de suite ${ }^{21} »$.

Les questions 11 à 19 portent sur l'arithmétique; nous y reviendrons plus en détail. Elles conduisent à la question 20, sur la quantité : est-elle divisible ? Suit une série de questions portant sur les objets de la géométrie (questions 21 à 34), notamment le continu, la grandeur, le point, la ligne, les figures, dont le cercle, et l'angle. Pour les définitions du point, de la ligne, de la figure et du cercle, Raoul le Breton se demande si elles sont bien posées (bene data), c'est-à-dire si, selon Aristote dans les Topiques (VI, 1), puis Porphyre dans l'Isagoge (III, 4), ces objets sont définis à partir de leur genre et de leur différence ${ }^{22}$.

Après la géométrie, Raoul en vient au comput (questions 35 à 39). Commentant la première phrase de l'introduction du De anni ratione de Sacrobosco, selon laquelle « le comput est la science qui considère les temps à partir des mouvements du Soleil et de la Lune ${ }^{23}$, Raoul commence par s'interroger sur le type de mouvements que l'on doit prendre en compte pour déterminer le temps, objet du comput. Puis à propos de la division du temps, Raoul se demande si l'atome (athomus) est une partie du temps ${ }^{24}$. Il en vient ensuite à la distinction entre jour naturel, composé de 24 heures, et jour artificiel, durée entre le lever et le coucher du Soleil ${ }^{25}$. Il s'intéresse ensuite au bissexte, vingt-neuvième jour ajouté au mois de février les années bissextiles. Enfin, Raoul se demande si on peut avoir des jours de fête mobiles.

21. Ms.P,f. 34rv : «Ad istam questionem dico quod inter omnes mathematicas arismetica est prior [...]. Item Boetius in sua arismetica sic probat quod arismetica sit prior geometria, quia illud quod habet notificare plures species magnitudinis vel omnes est prius magnitudine et notius. Modo numeri notificant plures species magnitudinis, sicut triangulus dicitur a 3, quadrangulus a 4 , pentagonus a 5 et sic de aliis. »

22. Ms. P, f. 38ra : « [...] omnis diffinitio bene data debet esse ex genere et differentia ».

23. Johannes de Sacrobosco, Libellus Ioannis de Sacro Bosco, de Anni ratione, seu ut vocatur uulgo, Computus Ecclesiasticus, Paris, 1550, f. 5r : «Computus est scientia considerans tempora ex Solis et Lunæ motibus. »

24. Le temps est divisé par Sacrobosco en jour, quadrant, heure, point, moment, uncia et atome (Libellus..., f. 5v).

25. Johannes de Sacrobosco, Libellus..., f. 6v : «Dierum autem alius Naturalis, alius Artificialis. Naturalis est 24 horarum, [...]. Dies vero Artificialis, est spatium temporis ab ortu Solis usque ad eius occasum [...]. » 
Le traité se termine par quatre questions sur la musique (questions 40 à 43), portant sur le début du De institutione musica de Boèce ${ }^{26}$. Raoul se demande d'abord, à propos de la musique, si c'est une science. On pourrait en douter du fait que la musique semble innée et qu'elle s'exprime de manières différentes selon les gens. Ayant répondu que la musique est bien une science, Raoul se demande si c'est une seule science. La question peut se poser si l'on considère que les objets de la musique sont d'une part les sons et d'autre part les nombres. Mais Raoul répond que la musique considère en fait les rapports entre les sons conçus comme des rapports de nombres, dont les mélodies sont des accidents ${ }^{27}$. Il s'interroge ensuite sur l'utilité de la musique. Il répond que « la musique est utile car est utile cette science grâce à laquelle les hommes grossiers et bestiaux sont domptés et rendus convenables ${ }^{28} \gg$. Finalement, Raoul s'enquiert de l'objet de la musique. Il redit que « le sujet propre de la musique est le rapport entre les sons ou le rapport entre les nombres reliés aux sons ${ }^{29} »$.

Dans le manuscrit de Bruxelles, à toutes ces questions font suites immédiatement deux autres, qui semblent donc appartenir au même ensemble. Dans la première, il est demandé si le sujet du De sphera est «l'univers ou quelque chose d'autre ${ }^{30}$ ». Dans le manuscrit de Paris, cette question est la dernière de la série de cinq questions considérées comme un commentaire du De sphera. La deuxième question en sus dans le manuscrit de Bruxelles est la suivante : « est-ce que les sciences mathématiques sont au premier degré de certitude ? ${ }^{31}$.

26. BoÈce, Traité de la musique, introduction, traduction et notes par C. MeYER, Turnhout, 2004.

27. F. Hentschel, Sinnlichkeit..., p. 295 : « Dicendum, quod musica est una scientia, [...], quia ibi consideratur de proportione sonorum sive de melodiis continentibus istam proportionem, unde istæ melodiæ ibi consideratæ sunt passiones, ergo, cum iste proportionales soni sive numeri sonorum proportionales sint quod unum subiectum, de quo probantur hic passiones, sicut sunt musicales consonantiæ, ita scientia erit una. » («On doit répondre que la musique est une seule science, [...] car y est considéré le rapport entre des sons ou les mélodies contenants ce rapport, de sorte que les mélodies considérées ici sont des accidents. Donc, puisque ces sons proportionnels ou ces nombres proportionnels de sons sont un seul sujet, pour lequel sont examinés ces accidents que sont les consonances musicales, ainsi la science sera une. »)

28. Ibid., p. 296 : «Dico, quod musica est utilis, quia illa scientia est utilis, per quam homines agrestes et bestiales domesticantur et propriantur. »

29. Ibid., p. 298 : «Credo, quod subiectum musicæ proprie sit proportio sonorum sive proportio numerorum relatorum ad sonos. »

30. Ms. B, f. 30r : « Consequenter queritur quid sit subjectum in spera, utrum universum vel aliquid aliud. »

31. Ms. B, f. 30rv : « Consequenter queritur utrum scientie mathematice sunt in primo gradu certitudinis. » 


\section{La forme Questio}

Comme son nom l'indique, les Questiones mathematice sont formées d'un ensemble de questions. La forme Questio est un genre littéraire issu de la dispute universitaire, qui suit un plan bien établi ${ }^{32}$. Prenons l'exemple de la première question qui concerne l'arithmétique, soit la question 11, qui demande si on peut avoir une science mathématique du nombre ${ }^{33}$. La question commence par quatre arguments quod non, c'est-à-dire des arguments qui prouvent que la réponse à la question posée doit être négative.

Ainsi, premièrement, il ne peut pas y avoir de science mathématique du nombre car « il ne peut pas y avoir de science mathématique de ce qui est sensible per se [c'est-à-dire connaissable par un ou plusieurs sens], or le nombre est de cette sorte, donc etc. ${ }^{34} \gg$. L'auteur reconnaît implicitement que le raisonnement est mis sous la forme d'un syllogisme dont la majeure est la proposition « il n'y a pas de science mathématique de ce qui est sensible per se », qui pour lui est évidente, et dont la mineure est la proposition « le nombre est sensible per se », qui est claire si l'on se réfère au livre II du $D e$ anima d'Aristote (II, 6, 418 a 20-23), « où il dit que la grandeur, le nombre, le mouvement etc. sont des sensibles communs. Or les sensibles communs sont sensibles per $s e^{35} \gg$.

À la toute fin de la question l'auteur répond aux quatre arguments quod non, qui sont contraires à la solution qu'il propose. À propos du premier il $\operatorname{dit}^{36}$ :

[...] lorsqu'il est dit que « il n'y a pas de science mathématique de ce qui est sensible per se » je réponds que c'est vrai pour ce qui est sensible per se et premier, mais qu'il peut y avoir une science mathématique de ce qui est sensible per se mais pas considéré comme premier, comme est le sensible d'une telle science. Or le nombre est un sensible per se, mais pas premier $[\ldots]$.

32. O. WeIJERS, La «Disputatio »...; EAD., « L'enseignement du trivium à la Faculté des arts de Paris : la "questio" », dans J. HAMESSE ed., Manuels, programmes de cours et techniques d'enseignement dans les universités médiévales, Louvain-la-Neuve, 1994, p. 57-74.

33. Ms. P, f. 34va : "Consequenter dictis queritur circa istas mathematicas in speciali et primo queratur circa arismeticam utrum de numero possit esse scientia mathematica. »

34. Ibid. : " Arguitur quod non quia de eo quod est per se sensibile non est scientia mathematica. Sed numerus est huiusmodi, quare etc. »

35. Ibid.: «Maior patet quia de eo quod est per se sensibile non est scientia mathematica. Minor patet ex secundo De anima ubi dicit quod magnitudo, numerus, motus etc. sunt sensibilia communia. Modo sensibilia communia sunt per se sensibilia.»

36. Ibid. : " Ad primam cum dicitur "de eo quod est per se sensibile non est scientia mathematica", dico quod verum est de eo quod est per se sensibile et primo, sed de eo quod est per se sensibile et non primo considerato, ut est sensibile de tali, potest esse scientia mathematica. Modo numerus est per se sensibilis, tamen non primo [...]. » 
En effet, le nombre est sensible du fait qu'il est conjoint à quelque chose d'autre qui est sensible et dont il peut être abstrait. Ainsi, on peut bien avoir une science mathématique du nombre.

Le deuxième argument quod non est le suivant ${ }^{37}$ :

[...] le sujet d'une science doit être unique, or le nombre n'est pas unique, donc etc. La majeure est claire car, comme une science est une, ainsi son sujet doit être un. La mineure est claire, car le nombre est essentiellement une multitude, or une multitude n'est pas un unum, donc etc.

Dans sa réponse, l'auteur réfute l'idée que la multitude ne serait pas un unum, car même si le nombre est bien une multitude, il est toutefois un selon son essence, à la fois quant à son espèce déterminée et quant à la définition commune aux espèces selon laquelle toute multitude est en soi indivisée mais divisée par autre chose («in se indivisa et ab alio divisa »). Ainsi, deux et trois sont bien, par essence, distincts entre eux et distincts des autres nombres ${ }^{38}$. Pour mieux comprendre cet argument on peut se rapporter à ce que dit Thomas d'Aquin dans l'article 1 (Utrum unum addat aliquid supra ens), de la Question XI (De unitate Dei) de la partie I de la Somme de théologie. Thomas y explique ${ }^{39}$ :

En effet, tout étant est soit simple, soit composé. Ce qui est simple est indivisé en acte et en puissance. Ce qui est composé n'a pas d'être tant que ses parties sont divisées, mais après qu'elles constituent et composent le composé. D'où il est clair que l'être de n'importe quelle chose consiste en une indivision.

37. Ibid. : « Item probo quod nulla scientia sit de numero, quia subjectum in scientia debet esse unum, sed numerus non est unus, quare etc. Maior patet quia sicut scientia est una, ita subjectum debet esse unum. Minor patet, quia numerus essentialiter est multitudo, modo multitudo non est aliquod unum, quare etc. »

38. Ms. P, f. 34vb : «Et cum dicitur quod "numerus non est unus", dico quod immo, saltem secundum genus. Et quando declaratur quod est multitudo, dico quod illa stant simul, scilicet quod numerus sit multitudo et quod sit unus secundum essentiam quantum ad determinatam speciem numeri et quantum ad unam rationem communem ad suas species, quia quelibet multitudo secundum se considerata est in se indivisa et ab alio divisa, sicut binarius est quedam essendi distincta a ternario et a quocumque alio. » ( "Et lorsqu'il est dit que "le nombre n'est pas un”, je réponds que c'est le contraire, du moins selon le genre. Et quand il est affirmé que c'est une multitude, je réponds que les deux vont ensemble, c'est-à-dire que le nombre est une multitude et qu'il est un selon l'essence, quant à l'espèce déterminée du nombre et quant à la définition unique commune à ces espèces, car n'importe quelle multitude considérée pour elle-même est en soi indivisée mais divisée par autre chose, comme deux est quelque mode d'être distinct de trois et de n'importe quel autre. »)

39. Thomas D'Aquin, Somme de théologie, Partie I, Question XI, article 1, [en ligne] http://www.thelatinlibrary.com/aquinas/q1.11.shtml [consulté le 17 juillet 2019] : « Nam omne ens aut est simplex, aut compositum. Quod autem est simplex, est indivisum et actu et potentia. Quod autem est compositum, non habet esse quandiu partes eius sunt divisae, sed postquam constituunt et componunt ipsum compositum. Unde manifestum est quod esse cuiuslibet rei consistit in indivisione. » 
Ainsi, le nombre 3 est un en tant que nombre, même s'il est aussi divisé en trois unités.

Troisième argument ${ }^{40}$ :

Toute science mathématique porte sur la quantité, mais le nombre n'est pas une quantité, donc etc. La majeure est claire à partir de ce qui précède. Preuve de la mineure, soit que le nombre n'est pas une quantité : en effet, à partir de choses qui ne sont pas des quantités n'est pas faite une quantité, or le nombre est fait à partir de choses qui ne sont pas des quantités, puisqu'il est obtenu à partir d'unités, donc il n'est pas une quantité.

Raoul répond que lorsqu'on dit qu'à partir de non-quantités on ne peut pas obtenir de quantité, c'est vrai pour la quantité continue, mais faux pour la quantité discrète, donc pour le nombre ${ }^{41}$.

Selon le quatrième argument ${ }^{42}$,

il n'y a pas de science de l'infini, or le nombre est de cette sorte, donc etc. La majeure est claire : l'infini, en tant qu'il est infini, n'est pas connaissable, selon ce qui est dit au premier livre de la Physique (I, 4, 187 b 7). La mineure est claire, car pour n'importe quelle espèce de nombre donnée on peut trouver un nombre plus grand, donc les nombres vont à l'infini par addition.

Raoul répond que ${ }^{43}$

lorsqu'il est dit « il n'y a pas de science de l'infini », c'est vrai pour autant que l'infini existe. Et lorsqu'il est dit que « le nombre est infini », je réponds qu'il n'y a pas de science du nombre pour autant qu'il aille à l'infini, mais il y a une science du nombre selon qu'il est réduit à ses espèces finies.

À la suite de ces quatre arguments quod non vient la seconde partie introduite pas la formule In oppositum, contenant les arguments prouvant le contraire, soit une réponse positive à la question posée. Ceux-ci se réduisent

40. Ms. P, f. 34va : «Item omnis mathematica est de quantite, sed numerus non est quantitas, ergo etc. Maior patet ex predeterminatis. Probatio minori, quod numerus non sit quantitas, quia ex non quantis non fit quantitas, sed umerus fit ex non quantis, quia est ex unitatibus, ergo non est quantitas. »

41. Ms. P, f. 34vb : « Ad aliam "omnis mathematica est de quantitate", conceditur. Et cum dicitur "numerus non est quantitas" falsum est. Et cum probatur "quia ex non quantis non fit quantitas", potest dici quod verum est continua sed discreta bene. »

42. Ms. P, f. 34va : "Item de infinito non est scientia, numerus est huiusmodi ergo etc. Maior patet : infinitum, secundum quod infinitum, est ignotum, sicut dicitur primo Physicorum. Minor patet quia, quacumque specie numeri data, adhuc contingit reperire maiorem numerum, unde numeri vadunt in infinitum per appositionem. »

43. Ms. P, f. 34vb : «Ad aliam, cum dicitur "de infinito non est scientia", verum est ut infinitum est. Et cum dicitur quod "numerus est infinitus", dico quod de numero, ut vadit in infinitum, non est scientia, sed de numero secundum quod reducitur ad species finitas est scientia. » 
à un appel aux autorités de Boèce et Jordanus, qui tous deux ont enseigné l'arithmétique et prouvent ainsi qu'il existe bien une science du nombre ${ }^{44}$.

Suit la réponse que Raoul propose à la question posée et pour laquelle il avance deux arguments ${ }^{45}$ :

À la question je réponds deux choses. Premièrement qu'il peut y avoir une science du nombre. Deuxièmement que celle-ci est l'arithmétique. On démontre ainsi la première, car on peut avoir une science mathématique de ce qui est hors de l'âme, a des accidents (passiones) et des propriétés démontrables et a des parties en lesquelles il peut être divisé et qui ne détermine de lui-même aucune qualité sensible.

Raoul explique alors que le nombre est bien de cette sorte, puis que l'objet de l'arithmétique est le nombre, dont elle donne la définition et les propriétés qu'il détaille, suivant à peu près ce que propose Boèce dans son Institution arithmétique ${ }^{46}$.

Toutes les questions suivent ce même plan : une série d'arguments quod non ou quod sic selon la réponse que soutiendra l'auteur à la question posée, l'appel aux autorités dans l'In oppositum, la réponse de l'auteur que l'on nomme parfois la determinatio et qui se termine par la réponse aux arguments donnés au début de la question et qui vont contre la solution adoptée par l'auteur.

\section{Le statut de l'unité et du nombre}

Nous allons maintenant examiner rapidement les questions 12 à 18 qui concernent le statut de l'unité et du nombre.

Tout d'abord, Raoul le Breton se demande si toutes les choses sont composées de nombres (question 12). Il s'agit ici de commenter la première proposition de l'Algorismus de Sacrobosco, rappelée au début de la question et selon laquelle « tout ce qui est apparu dès l'origine a été conçu au moyen des nombres ${ }^{47} \gg$. Raoul apporte une réponse négative à la question posée ${ }^{48}$ :

44. Ms. P, f. 34va : «In oppositum est Boetius et Iordanus qui de numero tradiderunt arismeticam. »

45. Ibid. : « Ad questionem dico duo. Primo quod de numero potest esse scientia mathematica. Secundo quod ista est arismetica. Primum sic declaratur quia de eo quod est ens extra animam et habet passiones et proprietates de eo demonstrabiles et partes in quas postet dividi et quod nullam qualitatem sensibilem sibi determinat, de omni tali potest esse scientia mathematica [...]. Modo numerus est tale ens [...].»

46. Bò̀CE, Institution arithmétique, texte établi et traduit par J.-Y. GUILlaumin, Paris, 1995.

47. Ms. P, f. 34vb : «Consequenter queritur circa Algorismum et primo de prima propositione ibi posita cum dicitur "omnia, que a primeva rerum origine processerunt, ratione numerorum formata sunt”, queritur utrum omnes res sint composite ex numeris. » Voir Johannes de Sacrobosco, Algorismus, Venise, 1501, f. Aii.

48. Ibid.: « Dico quod res non sunt constituende ex numeris, quia illud quod est posterius aliqua re non est principium constituendi eam, quia principia constituentia aliquam rem sunt 
Je dis que les choses ne sont pas constituées de nombres, car ce qui est postérieur à une chose n'est pas le principe qui constitue cette chose, car les principes qui constituent une chose sont antérieurs à cette chose. Or les nombres sont postérieurs aux substances, donc les substances ne sont pas composées de nombre.

Raoul s'interroge ensuite sur le statut du nombre. Il se demande si le nombre est bien une quantité (question 13) ${ }^{49}$. Raoul répond par l'affirmative $\operatorname{car}^{50}$

ce à quoi, en premier et per se, convient la définition de la quantité est une quantité, or au nombre convient la définition de la quantité qui est ou bien d'être divisible ou bien d'être ce par quoi quelque chose est formellement divisible.

En effet, le nombre est bien divisible en unités et le nombre est aussi ce par quoi quelque chose est divisible (on divise en deux parties, en trois parties, etc.).

Raoul en vient alors à la définition du nombre comme multitude composée à partir d'unités, donnée par Sacrobosco dans l'Algorismus (question 14) ${ }^{51}$. Il se demande si c'est une bonne définition, c'est-à-dire si le nombre est bien défini par son genre et sa différence. Là encore il faut distinguer deux manières de voir le nombre : soit comme quelque chose de divisible, soit comme ce par quoi quelque chose est divisible. Si l'on prend le nombre selon le premier mode, le genre du nombre est la multitude. Raoul remarque alors que ce genre ne s'applique pas aux seuls nombres. Il donne l'exemple du discours qui est une multitude formée de lettres et de mots. Il s'agit alors d'indiquer ce qui différencie le nombre du discours : c'est ce qui les compose. Le nombre est constitué d'unités, quand le discours est constitué de mots. Ainsi, dans la définition « le nombre est une multitude composée d'unités », «multitude » est le genre du nombre et « composé d'unités » est sa différence ${ }^{52}$. Si maintenant on considère le nombre comme

priora ista re. Modo numeri posteriores sunt substantiis ipsis, ergo saltem substantie non componuntur numeris. »

49. Ibid. : «[...] queratur utrum numerus sit quantitas ».

50. Ms. P, f. 35ra : « Dico quod numerus est quantitas quia illud cui primo et per se competit ratio quantitatis est quantitas, numerus est huiusmodi, quare etc. [...] Minor patet quia ratio quantitatis est vel esse divisibile vel illud quo aliquid est divisibile formaliter. »

51. Ibid. : «Consequenter queritur circa diffinitionem numeri qua declaratur "numerus est multitudo". » Voir Sacrobosco, Algorismus, f. Aii.

52. Ibid. : «Modo, si ponatur quod quantitas sit divisibilis in partes eiusdem rationis, tunc potest dici quod est bene data, quia ista diffinitio que est data de aliquo per genus et differentiam est bene data. Sed ista diffinitio data est de numero, ut est divisibilis in partes eiusdem rationis, quare etc. Maior patet. Minor patet quia, quando dico sic "numerus est multitudo", ibi est genus multitudo numeri, quia multitudo reperitur in numero et in multis allis, quia reperitur in oratione et in partibus orationis, ubi non est numerus. Etiam, cum dicitur "ex unitatibus agregata", istud ponitur ut differentia, quia illud est differentia numeri 
ce par quoi quelque chose est divisible, il s'agit d'un accident. Renvoyant à la Métaphysique d'Aristote (VII, 4, 1010b), Raoul explique que les définitions de l'accident diffèrent selon qu'il est abstrait ou concret, et « dans la définition d'un accident abstrait, on doit poser le sujet à la place de la différence et quelque chose de son genre à la place du genre ${ }^{53} \gg$. On peut rapprocher ici les réflexions de Raoul des explications que donne Thomas d'Aquin au chapitre $\mathrm{V}$ du De ente et essentia à propos des définitions des accidents ${ }^{54}$. Et c'est sans doute à Thomas, comme à Aristote, que Raoul reprend l'exemple de la définition de la camuserie comme courbure du nez (« simitas est nasi curvitas »). Le sujet de l'accident est le nez, et la courbure est quelque chose du genre de la camuserie. De la même manière, si l'on veut avoir une bonne définition du nombre abstrait conçu comme ce par quoi quelque chose est divisible, il faut qu'on ait un subjectum du nombre et quelque chose se rapportant au genre du nombre. Or « multitude » est le genre du nombre, et «composé à partir d'unités » renvoie au subjectum du nombre qui se compose de parties discrètes ou d'unités ${ }^{55}$.

per quod numerus separatur a quolibet alio. » (« Maintenant, si on pose que la quantité est divisible en parties de même raison, alors on peut dire que la définition est bien donnée, car une définition de quelque chose qui est donnée par le genre et la différence est bien donnée. Mais a été donnée au sujet du nombre cette définition qu'il est divisible en parties de même nature, donc etc. La majeure est claire. La mineure est claire car, quand je dis "le nombre est une multitude", ici la multitude est le genre du nombre, car la multitude se trouve dans le nombre et dans beaucoup d'autres choses, puisqu'on la trouve dans les discours et les parties des discours dans lesquels il n'y a pas de nombre. De plus, lorsqu'on dit "composé d'unités", c'est posé comme différence, car est différence du nombre ce qui différencie le nombre de quelque chose d'autre. »)

53. Ms. P, f. 35rb : « [...] in diffinitione accidentis in abstracto debet poni subiectum loco differentie et aliquid sui generis loco generis ».

54. Thomas D'Aquin, De ente et essentia, chap. 5, [en ligne] http://www. corpusthomisticum.org/oee.html [consulté le 17 juillet 2019] : « Et quia propriae passiones ex propriis principiis subiecti causantur, ideo subiectum ponitur in diffinitione eorum loco differentiae, si in abstracto diffiniuntur secundum quod sunt proprie in genere, sicut dicitur quod simitas est nasi curvitas. » (« Puisque les accidents propres sont causés à partir des principes propres du sujet, alors le sujet est posé dans leur définition à la place de la différence, si dans l'abstrait ils sont définis selon qu'ils sont propres au genre, comme lorsque l'on dit que la camuserie est la courbure du nez. »)

55. Ms. P, f. 35rb : «Ergo in diffinitione numeri in abstracto poni debet aliquid sui generis loco generis et aliquid sui subjecti loco differentie. Modo sic est in ista diffinitione quia, cum dicitur "numerus est multitudo" habeo genus numeri. Sed per hoc quod dicitur "ex unitatibus aggregata", ex unitatibus non materialiter sed effective, ex eo quod partium rei numerate quelibet est una, illud ponitur ratione subjecti numeri [...]. » («Donc, dans la définition du nombre dans l'abstrait, on doit poser quelque chose de son genre à la place du genre et quelque chose de son sujet à la place de la différence. Or on a bien cela dans la définition, car lorsqu'on dit "le nombre est une multitude", j'ai le genre du nombre. Mais lorsqu'il est dit "composé à partir d'unités", non pas matériellement mais de manière effective, du fait que n'importe laquelle des parties de la chose nombrée est une, ceci donne la nature du sujet du nombre. ») 
À la question suivante (question 15), Raoul se demande alors quelle relation il y a entre l'unité et la multitude ; plus précisément, il se demande si l'un (unum) et les multiples (multa) s'opposent ${ }^{56}$. La réponse dépend de la définition qu'on donne de l'un. Pour Raoul, il y a deux façons de le considérer : « [...] d'une manière selon qu'il entre dans la définition des nombres relativement à la multitude, d'une autre manière il peut être considéré comme quelque étant indivisé ou comme quelque étant divisé par autre chose ». Et Raoul précise que les deux dernières distinctions (étant indivisé, étant divisé par autre chose) ne sont pas réelles puisque « ce qui est divisé par autre chose est soi-même indivisé ${ }^{57}$. Nous avons vu en effet, à la question 11, que si le nombre est bien une multitude d'unités, il est toutefois un selon son essence. De la même manière, tout étant, même si on peut le diviser en parties, est un. Et Raoul ajoute ${ }^{58}$ :

Maintenant, si nous considérons l'un comme minimum relativement à la multitude, alors l'un et le multiple s'opposent relativement, car ils sont alors comme la mesure et le mesurable [...]. Mais si l'un et la multitude sont considérés de sorte que l'un exprime l'étant indivisé et la multitude l'étant divisé, je dis alors que l'un et la multitude s'opposent privativement [...], puisque le divisé et l'indivisé s'opposent privativement. [...] Et si l'un est considéré comme exprimant un étant divisé par autre chose, du fait qu'il est indivisé en soi mais divisé par autre chose, alors ils s'opposent relativement, comme le principe à ce qui est fondé [...].

56. Ms. P, f. 35rb : « Consequenter queritur utrum unum et multa opponantur.» On trouve la même question dans Thomas D'Aquin, Somme de théologie, Partie I, Question XI, article 2, [en ligne] http://www.thelatinlibrary.com/aquinas/q1.11.shtml [consulté le 17 juillet 2019].

57. Ms. P, f. $35 \mathrm{rb}$ : « Dicendum quod unum potest dupliciter considerari : uno modo secundum quod habet rationem numerorum respectu multitudinis, alio modo potest considerari ut est aliquod ens indivisum, [tertio] ut est aliquod ens divisum a quolibet alio, et quia ista duo includuntur in ratione unius, scilicet quod sit in se indivisum et divisum ab alio. »

58. Ms. P, f. 35rb : «Modo, si consideramus unum ut est minimum respectu multitudinis, sic unum et multa opponuntur relative, quia isto modo se habent sicut mensura et mensurabile [...]. éSed si unum et multitudo consideruntur ut unum dicit ens indivisum et multitudo ens divisum, ut sic dico quod unum et multitudo privative opponunturù (entre é et ù, corrigé à partir de B) [...], quia divisum et indivisum opponuntur privative. [...] Si autem unum consideratur ut dicit esse divisum ab alio quia est in se indivisum sed est divisum ab alio, sic opponuntur relative quia se habent sicut principium et principatum [...]. » Raoul propose ici une solution un peu différente de celle de Thomas d'Aquin. En effet, en réponse à la question «Utrum unum et multa opponantur », Thomas considère seulement deux modes pour l'unum: principe du nombre ou étant. Dans le premier cas, l'unum s'oppose au multa comme la mesure à ce qui est mesuré ; dans le second cas, l'unum s'oppose à la multitude sur le mode privatif, comme l'indivisé au divisé (Thomas d'Aquin, Somme de théologie, Partie I, Question XI, article 2 : «Respondeo dicendum quod unum opponitur multis, sed diversimode. Nam unum quod est principium numeri, opponitur multitudini quae est numerus, ut mensura mensurato: unum enim habet rationem primae mensurae, et numerus est multitudo mensurata per unum, ut patet ex X Metaphys. Unum vero quod convertitur cum ente, opponitur multitudini per modum privationis, ut indivisum diviso. ») 
On peut alors se demander si le nombre est un (question 16) ${ }^{59}$. La réponse est positive du fait que « ce qui peut être compris et signifié par une signification unique est unique ${ }^{60} »$. Or le genre du nombre est unique du fait que l'on a un seul genre là où une seule définition commune est donnée pour toutes les espèces, ce qui est bien le cas, puisque tout nombre est ce par quoi quelque chose est divisible en parties discrètes. On a aussi que chaque espèce de nombre est unique ${ }^{61}$.

Il faut alors se demander si le nombre a bien des espèces (question $17)^{62}$. Raoul répond que le nombre est bien un genre ayant des espèces. En effet, si l'on demande « qu'est-ce que trois ? (quid est ternarius ?), on répond que c'est un nombre. Donc le terme «nombre » est prédiqué « in quid » de plusieurs espèces, c'est-à-dire qu'au terme « nombre » est associé, via la question « qu'est-ce que c'est ? (quid est ?), plusieurs espèces de nombres différentes. C'est donc bien un genre ${ }^{63}$. Et les espèces qui composent le nombre diffèrent formellement, puisque la raison formelle de nombrer pour trois est différente de celle de nombrer pour quatre.

Finalement, il est demandé au sujet de l'addition si les nombres peuvent être composés entre eux (question 18) ${ }^{64}$. Raoul répond que c'est possible matériellement, mais pas formellement. C'est possible matériellement car, lorsqu'on additionne deux nombres, il y a autant d'unités matériellement dans la somme que dans chacun d'eux. Par contre c'est impossible formellement, comme le dit Aristote dans la Métaphysique $(\Delta 14,1020$ b 8-9) : deux trois ne sont pas six, formellement. En effet la

59. Ms. P, f. 35va : «Consequenter queritur utrum numerus sit unus. »

60. Ibid. : «Oppositum arguitur quia illud quod habet intelligi et significari una significatione [est unum, sed] (corrigé à l'aide de $\mathrm{B}$, « illud est » dans $\mathrm{P}$ ) numerus est huiusmodi, quare etc. »

61. Ibid. : «Dico quod numerus est unus, quicumque numerus sit, unde numerus in communi est unus secundum genus et numeri speciales sunt unum secundum speciem et particulares secundum numerum sicut hoc ternarius etc. » (« Je dis que le nombre est un, quel que soit le nombre, donc le nombre en général est un selon le genre et les nombres spéciaux sont un selon l'espèce et particuliers selon le nombre, comme ce trois etc. »)

62. Ibid. : « Consequenter queritur utrum numerus habeat species.»

63. Ibid. : «Dicendum primo quod numerus est genus et secundo quod habet plures species. Primum patet sic quia illud quod predicatur in quid de pluribus speciebus differt per formam et genus ad illas species, numerus est huiusmodi, ergo etc. Maior patet et minor quia, si queratur quid est ternarius, convenienter respondetur numerus, ergo predicatur in quid de istis. » («On doit répondre premièrement que le nombre est un genre et deuxièmement qu'il a plusieurs espèces. La première assertion est claire car ce qui est prédiqué "in quid" de plusieurs espèces diffère selon la forme et le genre de ces espèces, le nombre est de cette sorte, donc etc. La majeure est claire et la mineure aussi car, si on demande ce qu'est trois, on répond convenablement que c'est un nombre, donc il est prédiqué "in quid" de celles-ci. ») Pour la prédication « in quid», voir l'introduction de A. de Libera à PorpHYre, Isagoge, Paris, 1998, p. CVI-CXI.

64. Ms. P, f. $35 \mathrm{vb}$ : « Consequenter queritur circa additionem utrum numeri possint ex se invicem componi. » 
forme de six est une, c'est seulement six. Or, à partir de choses qui diffèrent formellement, on ne peut pas composer une troisième ${ }^{65}$. Ainsi, à partir de trois et quatre, on ne peut pas faire sept.

Les Questiones mathematice de Raoul le Breton sont un ouvrage de philosophie des mathématiques qui doit être étudié dans le contexte de l'enseignement à l'université de Paris, à la fin du XIII siècle. Ainsi, certaines réflexions générales sur les mathématiques font écho à ce que l'on trouve dans le De ortu scientiarum de Robert Kilwardby, rédigé vers 1250 à la faculté des arts de Paris ${ }^{66}$. Il est difficile à ce stade de notre recherche de dire si les réponses que Raoul apportent aux questions qu'il se pose sont originales. Frank Hentschel et Martin Pickavé avaient noté qu'on trouve des intitulés de questions identiques ou proches dans des commentaires de la Physique d'Aristote ${ }^{67}$. Ils avaient aussi signalé que Thomas d'Aquin était une source probable pour certaines questions ${ }^{68}$; nous avons pu le constater pour la question «Utrum unum et multa opponantur ». La dépendance de Raoul le Breton vis-à-vis de ses prédécesseurs ne peut être appréciée qu'à partir d'une comparaison fine des textes, rendue difficile par le fait que les sources des réflexions mathématiques de Raoul n'ont pas toutes été identifiées et que les textes auxquels il pourrait se rapporter ne sont disponibles que sous forme de manuscrits ou sont perdus.

Toutefois, on peut déjà souligner qu'un des intérêts de ces Questiones mathematice est que Raoul regroupe dans un même recueil et organise par sujet des questions que l'on trouvait jusqu'à présent, pour certaines, éparpillées dans les commentaires des ouvrages d'Aristote, notamment, ou

65. Ms. P, f. 36ra : «Dico ad questionem tria. Primo concedo quod numerus est quantitas sic particularis vel divisibilis quod numerus potest componi ex aliis materialiter. Secundo dico quod non potest componi ex hiis formaliter. [...] Primum declaratur quia, si accipiamus tria et tria, tot sunt unitates materialiter in istas, quot sunt in ipso senario. [...] Tamen ex numeris formaliter non constituitur numerus, quod apparet primo auctoritate Philosophi 5 Metaphysique, capitulo de quali. Dicit ibi quod bis tria non sunt sex. Verum est formaliter. Sed sex sunt vi. Solum senarius per formam senarii est senarius. » («Je réponds à la question trois choses. Premièremement, je concède que le nombre est une quantité particulière ou divisible de sorte que le nombre peut être composé à partir d'autres matériellement. Deuxièmement je dis qu'il ne peut pas être composé à partir d'eux formellement. [...] On démontre la première, car si nous prenons trois et trois, il y a autant d'unités dans ceux-ci matériellement qu'il y en a dans six. [...] Cependant un nombre n'est pas constitué formellement à partir de nombres, ce qui apparaît clairement d'abord selon l'autorité du Philosophe au livre V de la Métaphysique, au chapitre "de quali". Il y dit que deux trois ne sont pas six. C'est vrai formellement. Mais six sont VI. Seul six par la forme du six est six.»)

66. Robert Kilwardby, De ortu scientiarum, éd. A. G. Judy O. P., Toronto, 1976.

67. F. Hentschel et M. Pickavé, « Questiones mathematicales... », p. 630-632.

68. Ibid., p. 632-633. 
d'autres textes, de Raoul lui-même ou d'autres auteurs ${ }^{69}$. Malgré tout, la composition du texte n'est pas sans soulever des difficultés ${ }^{70}$ et le statut de ce texte est difficile à déterminer. $\mathrm{O}$. Weijers le classe parmi les introductions aux sciences, soulignant toutefois que, si certaines questions sont élémentaires et si leurs intitulés sont identiques à ceux que l'on trouve dans des recueils de questions servant à la préparation des examens, d'autres questions sont plus techniques ${ }^{71}$. Frank Hentschel et Martin Pickavé contestent le caractère introductif du traité, tout en reconnaissant que les parties sur l'arithmétique et le comput peuvent être vues comme des introductions à la lecture des traités de Sacrobosco ${ }^{72}$. Quoi qu'il en soit, si ces Questiones sont typiques des textes produits à la faculté des Arts à cette époque, il est impossible de savoir si elles sont le reflet d'un véritable enseignement ou si elles résultent d'un intérêt propre de Raoul le Breton pour ces sujets, qui se serait alors proposé de les rassembler dans un même texte.

Comme l'ont montré F. Hentschel et M. Pickavé, d'autres maîtres ès arts de l'université de Paris ont proposé des Questiones mathematice, à la suite de Raoul le Breton, dans les années 1323-1347. On trouve ainsi des questiones anonymes dans un manuscrit de Londres et une compilation anonyme de questiones de quatre maîtres, dans un manuscrit de Munich ${ }^{73}$. Là encore le lien entre la production de ces questiones et l'enseignement se pose ${ }^{74}$.

Sabine Rommevaux-Tani - CNRS - Université de Paris, Université Paris 1, SPHERE, UMR 7219

69. C'est le cas pour la question de savoir si la quantité est divisible, qui est traitée par Raoul dans les Questiones mathematice, faisant la charnière entre les questions sur l'arithmétique et les questions sur la géométrie, mais aussi, dans un autre contexte, dans ses Questiones super predicamenta et dans ses Questiones super librum sex principiorum (je remercie Costantino Marmo qui m'en a fait la remarque).

70. F. Hentschel et M. Pickavé remarquent ainsi que dans le manuscrit de Paris un espace blanc de plusieurs lignes suit la question sur l'angle, dernière des questions sur la géométrie, ce qui pourrait laisser penser que le texte s'arrête ici ou que le texte est incomplet. Or l'explicit vient plus loin, à la fin des questions sur la musique et présente l'ensemble des questions qu'il clôt comme un tout attribué à Raoul le Breton. Ils remarquent aussi que la musique n'est pas mentionnée dans l'explicit. Ils s'étonnent enfin de l'absence de questions sur le De sphera, alors que ce traité est cité dans le prologue (F. Hentschel et M. Pickavé, «Questiones mathematicales..., p. 629). Cette absence pourrait justifier l'ajout de questions sur ce sujet dans le manuscrit de Paris, à la suite des Questiones de Raoul le Breton.

71. O. WeIJers, La « Disputatio »..., p. 98 .

72. F. Hentschel et M. Pickavé, «Questiones mathematicales...», p. 634.

73. Ce recueil a été édité par G . Dell'Anna dans Theorica mathematica et geometrica medievalia, Galatina, 1992.

74. F. Hentschel et M. Pickavé, « Questiones mathematicales...», p. 618-634. 


\section{L'unité et le nombre dans les Questiones mathematice de Raoul le Breton}

Raoul le Breton ou Radulphus Brito, maître ès arts à l'université de Paris, produit dans les dernières années du XIII ${ }^{e}$ siècle un traité de philosophie des mathématiques, qu'il organise sous formes de questiones, typiques des textes universitaires produits à cette époque. Après une présentation générale de ces Questiones mathematice, nous donnerons un aperçu des réflexions de Raoul sur l'unité et le nombre.

arithmétique, enseignement, philosophie des mathématiques, Radulphus Brito, Raoul le Breton, université de Paris

\section{Unity and Number in the Questiones mathematice by Radulphus Brito}

In the last years of the thirteenth century, Raoul le Breton or Radulphus Brito, Master of Arts at the University of Paris, produced a treatise on the philosophy of mathematics, which he organized in the form of Questiones, typical of university texts produced at that time. After a general presentation of these Questiones mathematice, I will give an overview of Raoul's reflections on unity and number.

arithmetic, philosophy of mathematics, Radulphus Brito, teaching, University of Paris 
